\section{VIVIENDAS DE EMERGENCIA: REFLEXIONES A PARTIR DE LA EXPERIENCIA DEL TERREMOTO DEL 27F ${ }^{1}$}

Rose Marie Garay Moena²

\section{EMERGENCY HOUSING: REFLECTIONS ON THE EXPERIENCE OF THE 27F EARTHOUAKE ${ }^{1}$}

Rose Marie Garay Moena²

\section{Resumen}

Tras la experiencia de los terremotos, 27F, 2010, 1 de abril 2014 en el norte grande e incendio del 12 de abril en Valparaíso, se evidencian, una vez más, carencias graves en la preparación y capacitación del país frente a desastres. Específicamente, la baja calidad de las viviendas de emergencia obedece a aspectos legislativos deficitarios que operan en el momento de su entrega por parte del Estado en situaciones de desastres socio-naturales o de vulnerabilidad social. En este apartado se analizan los cuerpos legales y normativos conducentes a responder frente a la emergencia, y en cuanto a la calidad de las viviendas entregadas. Se concluye

\begin{abstract}
The experiences of the earthquakes that shook central and northern Chile on February 27, 2010 and April 1, 2014 respectively, and the great fire that affected Valparaiso on April 12, 2014, reveal serious shortcomings in terms of disaster preparedness and training. Specifically, the low quality of emergency dwellings reflects the poor legislative aspects that operate within the context of socio-natural disasters or social vulnerability. This paper analyzes the statutes and regulations intended to cope with emergency situations and address the quality of housing. The resrach concluded that there is no legal regime that
\end{abstract}


que no existe un régimen legal que comprometa calidad bajo criterios mínimos de habitabilidad, quedando ello, sólo supeditado a instrucciones de orden económico, como fijación de precios. El aseguramiento de calidad de la vivienda, así como procedimientos frente a desastres para la habilitación de aldeas o campamentos que aseguren condiciones mínimas, requiere de planificación, reglamentación e implementación para evitar improvisaciones. A pesar de estar disponibles otras normativas tales como la evacuación en emergencias, ellas no han resultado suficientemente operativas. Se espera que la promulgación de la ley de protección civil resulte un instrumento más efectivo que incorpore a las viviendas de emergencia.

PALABRAS CLAVE: VIVIENDA DE EMERGENCIA, SISTEMA NACIONAL DE PROTECCIÓN CIVIL, ORDENANZA GENERAL DE URBANISMO Y CONSTRUCCIÓN

Fecha de recepción: 08.07.13

Fecha de aceptación: 20.01.15

1 Este texto es resultado de la investigación FONDEF I+D D09I1058 Conicyt. Co-investigadores: Ricardo Tapia, Jorge Larenas, Francis Pfenniger; colaboradores: Mauricio Toledo y Paulina Vera.

2 Chile, Profesora asociada, Departamento Ingeniería en Maderas y sus Biomateriales, Facultad de Ciencias Forestales y de la Conservación de la Naturaleza, Universidad de Chile. Correo electrónico: rgaray@uchile.cl

214 revista invi № 83 / Mayo 2015 / Volumen № 30: 213-221 ensures the provision of decent housing; this being a task subject to economic guidelines such as a preocupation on pricing. Ensuring the provision of quality housing and procedures aimed at coping with disasters and the establishment of proper settlements and emergency camps demands a planning process, regulations and implementation in order to avoid improvisation. There are other regulations such as those related to emergency evacuation; however, they have been proven partially operative. It is hoped that the enactment of the Civil Protection Act provides an effective instrument for the incorporation of emergency housing.

KEYWORDS: EMERGENCY HOUSING， NATIONAL SYSTEM FOR CIVIL PROTECTION, GENERAL ORDINANCE ON URBAN PLANNING AND CONSTRUCTION

Received: 08.07.13

Accepted: 20.01.15

1 The paper is the result of the FONDEF I+D Conicyt research D09I1058. Co-researchers: Ricardo Tapia, Jorge Larenas, Francis Pfenniger; collaborators: Mauricio Toledo y Paulina Vera.

2 Chile. Forestry Engineer, Ms in Forestry Sciences. Associate professor in the Department of Wood Engineering and Biomaterials, Faculty of Forestry Sciences and Nature Preservation, University of Chile. Email: rgaray@uchile.cl

OPINION: Emergency housing: reflections on the experience of the $27 \mathrm{~F}$ earthquake / Rose Marie Garay Moen 


\section{Los hechos: respuesta a la emergencia con mediaguas}

Para todo el país resulta impresentable que cada vez que ocurre un desastre natural o antrópico, nos tengamos que enfrentar a la poca previsión en el accionar frente a las emergencias. En ciertas materias, se carece de planes de contingencia, mientras que en otras, pese a existir, se falla en la implementación. Si se observa el problema desde cualquier arista, se constata la necesidad urgente de una planificación multi-institucional, con todos los organismos alineados tras un gran programa, del tipo Sistema Nacional de Protección Civil, pero que funcione. La experiencia muestra que existen buenas ideas, incluso en la fase de preparación y de alerta temprana, pero aquellas fracasan por la falta de implementación práctica de todos los eslabones de la gestión.

En el campo específico de las viviendas de emergencia, en Chile históricamente se ha enfrentado el problema con "mediaguas" -viviendas de emergencia precarias, construidas en base a paneles prefabricados de madera y que no cumplen las condiciones mínimas de seguridad ni habitabilidad. La raíz de muchos de los problemas de las alternativas de vivienda de emergencia se explica

OPINIÓN: Viviendas de emergencia: reflexiones a partir de la experiencia del terremoto del 27F / RoseMarie Garay Moena por disposiciones legales (o la ausencia de éstas) que permiten la entrega de estas viviendas en situaciones de vulnerabilidad social y de desastres naturales. Esto ocurre porque no existen estándares establecidos para responder con viviendas de mejores características técnicas ${ }^{2}$. La regulación actual sólo establece límites presupuestarios para el gasto unitario en que puede incurrir el Estado para cubrir necesidades de vivienda por emergencia. Por el contrario, ello sí sucede en el caso de las viviendas sociales -de carácter definitivo-, cuyo marco regulatorio se establece en la Ordenanza General de Urbanismo y Construcción del Ministerio de la Vivienda y Urbanismo (MINVU) ${ }^{3}$. Sin embargo, la naturaleza provisoria de las viviendas de emergencia las exime de tales regulaciones.

\section{Origen: disposiciones legales sobre viviendas durante emergencias}

Si se reconstruye el modus operandi implementado tras el 27F del 2010, los hechos acontecieron de acuerdo a las siguientes disposiciones legales:

i. Se aplicó la Ley 16.282 del año 1965, que fija disposiciones para casos de sismos o catástrofes.

CCHC, 2010

OGUC,2007 
ii. La ley $\mathrm{N}^{\circ} 16.282$ fue modificada por Decreto 104 del año 1977, fijando disposiciones para casos de sismos o catástrofes y estableciendo normas para la reconstrucción de la zona afectada por el sismo del 28 de marzo de 1965, proveniente del Ministerio del Interior, que fija texto refundido, coordinado y sistematizado del Título I de la Ley 16.282, determinando las disposiciones permanentes para casos de sismos o catástrofes.

Un resumen de los contenidos de algunos de los artículos permite percibir que los cuerpos legales se redactaron para hacer funcionar el aparato gubernamental en caso de desastres. Por tanto, el problema está en cómo se implementan estas disposiciones legales, es decir, en etapas posteriores de la cadena de reacción. En los párrafos que siguen, se resumen las indicaciones sobre las viviendas de emergencia que describen los artículos indicados, identificando los problemas que se generan y sugiriendo mejoras.

\section{Toma de decisiones y normas de excepción}

En el Art. 1 de la ley $N^{0}$ 16.282, se señala que en el caso de producirse un sismo o catástrofe en el país, que dañe a las personas o a los bienes, el Presidente de la República dictará un Decreto Supremo señalando las comunas afectadas. A su vez el Art.
3 indica que el Presidente de la República podrá, por Decreto Supremo fundado, dictar normas de excepción del Estatuto Administrativo, de las Leyes Orgánicas de los Servicios Públicos, de las instituciones autónomas o semifiscales, para resolver problemas de las comunas afectadas. De forma que una de las normas de excepción a las que se puede acceder, es la designación de autoridades y determinación de sus atribuciones o facultades. Por lo tanto, las normas de excepción son herramientas útiles que permiten enfrentar problemas específicos, y que posibilitarían brindar mayor autonomía y antelación a las acciones que debiera implementar el Plan Nacional de Protección Civil, por mencionar alguno de los planes que se necesitaría que funcione.

\section{Aportes extrapresupuestarios y mecanismo de asignación}

Por su parte, el Art. 9 señala que las Municipalidades podrán pedir aumentos en sus presupuestos en razón de la catástrofe. Así, el Fisco puede entregar aportes extraordinarios a las Municipalidades, con cargo a lo determinado por el Art. 72 $\mathrm{N}^{\circ} 10$ de la Constitución (DFL Na 1 , 2006) como fondo para la calamidad pública, y otros que designen las leyes para aportes extraordinarios, para que la Municipalidad pueda disponer de recursos materiales que paleen el estado catastrófico. Sin 
embargo, la supervisión del buen uso de los fondos debería estar protegida por instancias predefinidas de forma explícita que fijen de forma estricta para qué y cómo se usarían estos recursos, acotándolos para ir en auxilio de quienes estén afectados por la emergencia. Esto último se enlaza con la pésima experiencia en términos de ficha de clasificación social o ficha CASEN utilizadas. Tales fichas estaban desactualizadas y eran poco rigurosas, lo que permitió el abuso de gente sin escrúpulos a las que se les asignaron viviendas de emergencia sin necesitarlas. Esto perjudicó a otras personas que sí lo requerían. Como muchas otras veces, se comprueba que la intención de la Ley es buena, pero la falta de previsión y la baja prolijidad en el trabajo previo conllevan finalmente a un mal uso del instrumento.

\section{Respuesta inicial a catástrofes naturales}

En el país, instituciones como las Fuerzas Armadas, Carabineros y Bomberos son las que están mejor preparadas para enfrentar los desastres. Esta situación está descrita en el Art. 20, donde se señala que el Ministro del Interior y el de Defensa Nacional son responsables de la elaboración de un plan tendiente a obtener que las Fuerzas Armadas y el Cuerpo de Carabineros desarrollen un programa que abarque los puntos de: a) Preparar un plan orgánico para las emergencias que se produzcan; b) Programar la coordinación de los recursos humanos y materiales de los servicios públicos y de las instituciones asistenciales. Luego, los organismos encargados de iniciar los planes de acción serían el Ministerios del Interior y el de Defensa Nacional, y por tanto son los primeros responsables. El Art. 21 señala en forma explícita que es el Ministerio del Interior el que tendrá a su cargo la planificación y coordinación de las actividades que señala esta Ley y la atención de sismos y catástrofes.

\section{Municipios como coordinadores}

Un apoyo ineludible y lógico está contemplado en el Art. 22 que establece la constitución de un Comité Comunal de Emergencia. Entre sus funciones más importantes está la de disponer de medidas que deban adoptarse de inmediato y que no hayan sido tomadas por la autoridad competente. Este Comité propone a la autoridad medidas en interés de la comunidad y participa en la distribución de ayuda. Sin embargo, el aparato gubernamental está consciente de las enormes disparidades de criterios, recursos y condiciones que proyectan al infinito las posibilidades de conciliar tales criterios, en aspectos relevantes y prioritarios como el establecimiento a priori de infraestructura y equipamiento para implementar refugios, de forma de 
evitar improvisaciones como el uso de escuelas o gimnasios, sin esta implementación mínima. En este sentido, hay acuerdo en algo: falta casi todo por hacer para la implementación de estas acciones bajo criterios de planificación de riesgos y al parecer resulta casi imposible intervenir en el área de acción de las alcaldías. Así, para efectos de la ciudadanía, esta característica podría jugar a favor o en contra, según sea el grado de responsabilidad y preparación que cada Municipio asuma. Es decir, los chilenos estamos expuestos no sólo a los desastres por amenazas naturales sino al azar en términos de si los municipios están preparados para enfrentarlos. Esto último dista mucho de ser un escenario adecuado, porque consolida las diferencias sociales y las divisiones entre las personas.

\section{Planes de reconstrucción}

Se establece que dentro de un plazo de 180 días a contar de la publicación del Decreto que declara la situación de emergencia, la Oficina Nacional de Planificación -que ya no existe con ese nombre-y los Organismos que integren el Sistema Nacional de Planificación, deben presentar al Presidente los planes regionales de reconstrucción y desarrollo para cada una de las regiones afectadas, (Art. 24.) lo que gatilla el accionar de gobernadores provinciales e intendencias regionales. Sin embargo, a vista de las personas, poco se entiende su accionar si finalmente son los Municipios los que tienen las atribuciones operativas. Por lo tanto, si el gobierno pide los planes regionales resulta obvia la necesidad de un trabajo cooperativo, pero que sin mediar el enlace legal que establezca esta conexión no parece ser tan expedito y poco es el avance que se ve en materia de implementación práctica de tales planes.

\section{Agilización de tramitación de viviendas}

Más directamente relacionado con las viviendas, se debe mencionar que el Art. 25 hace referencia a que no es necesario un arquitecto que avale los planos en las viviendas individuales, especificaciones y demás antecedentes tipos proporcionados por el MINVU cuando se trate de viviendas prefabricadas, o al plano original de las viviendas provenientes de la industria fabricante inscrita en el Registro de Productores de Viviendas y Construcciones industrializadas y prefabricadas que lleva el mismo MINVU. Tanto planos como especificaciones y otros, serán puestos a disposición de la Municipalidad por el MINVU. En este sentido, deslinda responsabilidad a otras instancias para el caso general de viviendas prefabricadas, sin entrar en materia específica respecto a las viviendas de emergencia. 


\section{Exigencias extraordinarias a viviendas}

El Art. 26 señala por primera vez la incidencia puntual que tiene el Ministerio de Vivienda y Urbanismo para efectos de la construcción, reconstrucción y reparación de edificios, y su incidencia en cuanto a los planos, especificaciones, para todo lo cual tiene un plazo para determinar exigencias extraordinarias. Señala que dentro del plazo de 30 días desde la entrada en vigencia del Decreto que declara las comunas afectadas por el sismo, el MINVU establecerá las exigencias extraordinarias a que deberán ajustarse la construcción, reconstrucción y reparación de edificios, o demolición de ellos.

\section{Foco en contención del gasto}

Tal como se mencionó previamente, desde el Estado hay más preocupación por la protección y regularización del gasto que por la calidad o requisitos exigibles a las viviendas. Ello se demuestra en el Art. 45 en donde se señala que el examen de cuentas de los dineros utilizados vía Ministerio del Interior son apreciados por el Contralor General de la República, en este caso puntual se señala la apreciación en conciencia de la prueba por falta de algún documento que respalde el gasto.

\section{Vivienda de emergencia, un tema de costo}

Finalmente, en la búsqueda del soporte legal de las viviendas de emergencia aparece la presente estructura legal: Mediante el Decreto Ley 2552 del año 1979, proveniente del MINVU, deroga el Decreto Ley $\mathrm{N}^{\circ} 1088$, de 1975, y transfiere al MINVU los programas de "Viviendas Sociales"; modifica el Decreto Ley No 1519, de 1976, define las "viviendas de emergencia" y señala competencia de la Oficina Nacional de Emergencia del Ministerio del Interior (ONEMI). En el Art. 5 señala que ONEMI no podrá construir o encomendar construir otro tipo de viviendas que no sean las de emergencia. Esta es una definición muy ambigua, no señalando el ámbito de injerencia que en efecto tiene, por tanto, el MINVU a través del Servicio de Vivienda y Urbanismo (SERVIU), no tendrá obligación de supervisar este tipo de vivienda y el tema de la calidad de éstas queda sin abordar de forma explícita. Solamente se define la vivienda de emergencia, como aquella cuyo valor no excede el equivalente en pesos de 30 UF (aprox. US\$1.350), puestas en la bodega de la ONEMI. Si el tamaño de la familia afectada excede las cinco personas, se agregan 5 UF (aprox. US\$225) por cada persona que exceda dicho número.

Será por el Ministerio del Interior, a través de Decretos Supremos, que se reglamente la forma de 
asignar, dar en comodato o arrendar las construcciones hechas para soluciones habitacionales de emergencia. Será función de la ONEMI, en coordinación con la Municipalidad, atender los casos de emergencia habitacional, derivados de hechos tales como terremotos o calamidades semejantes, pero no tendrá la ONEMI la facultad de adquirir inmuebles ni terrenos. Se reitera, por tanto la ambigüedad de la función en el verbo "atender", al que no se le expresan sus límites.

\section{Conclusión}

Por lo anteriormente expuesto, es que el establecimiento de criterios técnicos mínimos de habitabilidad para las viviendas de emergencia, y paralelamente, la existencia de un reglamento general que rija tanto a las viviendas como a su contexto de instalación, podrán aportar a un mejoramiento operativo frente a desastres. Aunque falta que ambos sean incluidos en el aparato legislativo, tal como contenidos en una norma chilena obligatoria de requisitos para viviendas de emergencia, o bien, bajo articulado de la Ley de protección civil u otra forma que se pudiese acordar, pero que garantice la regulación de este importante tema.

\section{Bibliografía}

CÁMARA Chilena de la Construcción. 10 claves sobre el terremoto y la calidad de las viviendas. CChC. 15 de marzo de 2010. [Fecha de consulta: 19 de abril de 2010]. Disponible en: http://www.cchc.cl/ noticias/detalle. asp?id=4363

\section{Normas citadas}

\section{CóDIGO Civil.}

CóDIGO Orgánico de Tribunales.

D. F. L. $N^{0} 1$, Ministerio de Interior, 9 de mayo de 2006, Fija texto refundido, coordinado y sistematizado de la ley $\mathrm{N}^{\circ} 18.695$, Ley Orgánica Constitucional de Municipalidades. Diario Oficial 26 de julio de 2006

LEY N 19.496, Establece Normas sobre Protección de los Derechos de los Consumidores. Diario Oficial, 7 de marzo de 1997.

D.L. No 1289, de 1975, Ley Orgánica de Municipalidades (derogada). Diario Oficial, 14 de enero de 1976.

DECRETO con Fuerza de Ley $\mathrm{N}^{\circ} 458$, Ministerio de Vivienda y Urbanismo, de 18 de diciembre de 1975, 
Ley General de Urbanismo y Construcciones. Diario Oficial, 13 de abril de 1976.

LEY N 19.472, Modifica el D.F.L. No 458, Ley General de Urbanismo y Construcciones, estableciendo Normas relativas a la Calidad de la Construcción. Diario Oficial, 16 de septiembre de 1996.

LEY N ${ }^{0}$ 20.016. Modifica Normas del Decreto con Fuerza de Ley No 458, de 1975, Ley General de Urbanismo y Construcciones, relativas a la calidad de la construcción. Diario Oficial, 27 de mayo de 2005.

DECRETO Supremo $N^{\circ} 47$, Ministerio de Vivienda y Urbanismo, de 16 de abril de 1992, Fija Nuevo Texto de la Ordenanza de la Ley General de Urbanismo y Construcciones. Diario Oficial, 5 de junio de 1992. 